\section{(9) \\ BRAZIULIAN JOURNAL \\ OF MEDICAL AND BIOLOGICAL RESEARCH}

www.bjournal.com.br
ISSN 0100-879X

Volume 45 (7) 565-680

July 2012

CLINICAL INVESTIGATION

Braz J Med Biol Res, July 2012, Volume 45(7) 625-631

doi: 10.1590/S0100-879X2012007500071

Trans fatty acid intake is associated with insulin sensitivity but independently of inflammation

C.T. Angelieri, C.R. Barros, A. Siqueira-Catania and S.R.G. Ferreira

The Brazilian Journal of Medical and Biological Research is partially financed by

\section{욛NPq}

Ministério

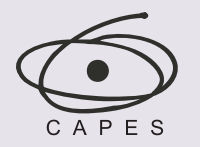

Ministério da Educação

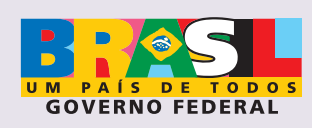

DTFAPESP

Institutional Sponsors

๑ SHIMADZU UNICAMP

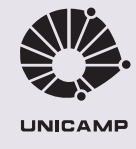

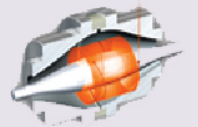

1DI Associaçăo Explore High - Performance MS Orbitrap Technology In Proteomics \& Metabolomics analitica $\underset{\text { analiticaweb.com.br }}{\text { Thermo }}$
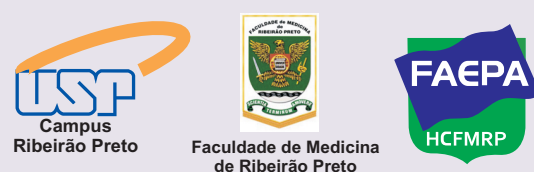


\title{
Trans fatty acid intake is associated with insulin sensitivity but independently of inflammation
}

\author{
C.T. Angelieri, C.R. Barros, A. Siqueira-Catania and S.R.G. Ferreira \\ Departamento de Nutrição, Faculdade de Saúde Pública, Universidade de São Paulo, São Paulo, SP, Brasil
}

\begin{abstract}
High saturated and trans fatty acid intake, the typical dietary pattern of Western populations, favors a proinflammatory status that contributes to generating insulin resistance (IR). We examined whether the consumption of these fatty acids was associated with IR and inflammatory markers. In this cross-sectional study, 127 non-diabetic individuals were allocated to a group without $I R$ and 56 to another with IR, defined as homeostasis model assessment-IR (HOMA-IR) $>2.71$. Diet was assessed using 24-h food recalls. Multiple linear regression was employed to test independent associations with HOMA-IR. The IR group presented worse anthropometric, biochemical and inflammatory profiles. Energy intake was correlated with abdominal circumference and inversely with adiponectin concentrations $(r=-0.227, P=0.002)$, while saturated fat intake correlated with inflammatory markers and trans fat with HOMA-IR $(r=0.160, P=0.030)$. Abdominal circumference was associated with HOMA-IR ( $r=0.430, P$ $<0.001)$. In multiple analysis, HOMA-IR remained associated with trans fat intake $(\beta=1.416, P=0.039)$ and body mass index $(\beta=0.390, P<0.001)$, and was also inversely associated with adiponectin $(\beta=-1.637, P=0.004)$. Inclusion of other nutrients (saturated fat and added sugar) or other inflammatory markers (IL-6 and CRP) into the models did not modify these associations. Our study supports that trans fat intake impairs insulin sensitivity. The hypothesis that its effect could depend on transcription factors, resulting in expression of proinflammatory genes, was not corroborated. We speculate that trans fat interferes predominantly with insulin signaling via intracellular kinases, which alter insulin receptor substrates.
\end{abstract}

Key words: Insulin resistance; Inflammation; Fatty acids

\section{Introduction}

High energy and high fat intake characterizes the diet of numerous Western populations. Particularly, the deleterious role of excessive saturated fatty acid (SFA) intake on the cardiovascular system has been consistently demonstrated. When combined with physical inactivity, these habits contribute to the accumulation of body fat, which generates a pro-inflammatory status. Several lines of evidence support the notion that SFA intake is the main trigger of inflammation in white adipose tissue (1).

Adipocytes play a major role in energy balance, immune function and homeostasis of glucose and lipid metabolism (2). Excessive SFA consumption leads to adipocyte hypertrophy and dysfunction, inducing the secretion of inflammatory factors, which results in a status of chronic low-grade inflammation (3) and is the pathophysiological basis for metabolic and cardiovascular diseases (2). In addition to SFA, trans fatty acid intake is also associated with metabolic disturbances (4).

Hypertrophic adipocytes and macrophages are responsible for the increased expression of pro-inflammatory genes and secretion of cytokines such as interleukins and tumor necrosis factor alpha (TNF- $\alpha$ ). The latter was the first adipocytokine found to be directly associated with insulin resistance $(5,6)$.

Obesity-induced cytokine secretion (TNF- $\alpha$, interleukins) promotes an increase in hepatic synthesis of C-reactive protein (CRP), whose concentration has been used to assess the risk of cardiovascular disease as well as type 2 diabetes mellitus. Conversely, adiponectin concentration has been associated with protective cardiometabolic effects (2). It is possible that the determination of circulating levels of TNF- $\alpha$, interleukins, CRP, and adiponectin may enhance the knowledge of the pathophysiological events linking SFA consumption and insulin resistance induction.

Correspondence: S.R.G. Ferreira, Departamento de Nutrição, Faculdade de Saúde Pública, USP, Av. Dr. Arnaldo, 715, 01246-904 São Paulo, SP, Brasil. Fax: +55-11-3061-7701. E-mail: sandrafv@usp.br

Received December 2, 2011. Accepted April 24, 2012. Available online May 11, 2012. Published July 2, 2012. 
It was previously found that SFA stimulate intracellular insulin signaling via the nuclear factor- $\mathrm{KB}(\mathrm{NF}-\mathrm{kB})$, negatively influencing the translocation of glucose transporters to the plasma membrane (3).

In addition to the deleterious impact of dietary SFA on metabolism and the cardiovascular system $(4,7)$, prospective studies have reported an association of trans fatty acid intake with increased risk of type 2 diabetes mellitus (8). In contrast, diets rich in unsaturated fatty acids - such as the Mediterranean diet - have been shown to have beneficial effects against cardiovascular events (9). Such evidence elucidating the role of the consumption of different fatty acids in insulin sensitivity is relevant to orient health policies for populations at cardiometabolic risk (1). Another lifestyle factor of relevance for cardiometabolic homeostasis is physical activity. Inactivity is considered to be a major cardiovascular risk factor, while regular physical activity improves glucose and lipid metabolism $(3,4,10,11)$.

Since the main causes of mortality are adiposity-related diseases (12-14), it is desirable to understand how daily habits influence their pathophysiologic chain. Little data are available concerning the association of fatty acid intake with metabolic disturbances, adjusted for physical activity, mediated by inflammation and insulin resistance, in Brazilian population samples. We hypothesized that, similar to the role of SFA in insulin resistance, trans fatty acid intake may also contribute to the deterioration of insulin sensitivity, which may be mediated by proinflammatory markers. Therefore, the objective of the present study was to assess the associations of saturated or trans fatty acid intake with insulin resistance.

\section{Material and Methods}

This cross-sectional study included individuals at high cardiometabolic risk, selected for an 18-month prevention program of type 2 diabetes mellitus, conducted at the Health Center of the Faculdade de Saúde Pública, Universidade de São Paulo, Brazil. The local Research Ethics Committee approved the research protocol and all participants gave written informed consent. Of 438 individuals screened between 2008 and 2009, 230 were eligible and 183 agreed to participate. Among those who did not agree to participate there was a predominance of men; however, non-participants did not differ from participants in terms of sociodemographic, anthropometric or metabolic variables. Their basal dietary, clinical and laboratory data were analyzed. Inclusion criteria were adults aged 18 to 69 years with prediabetic conditions (impaired glucose tolerance or impaired fasting glycemia) (15) or with metabolic syndrome without diabetes, defined according to the International Diabetes Federation (16). Individuals with a medical history of neurological or psychiatric disturbances, thyroid, liver, renal, and infectious diseases were excluded.

\section{Data collection}

Trained staff collected dietary, physical activity and clinical data. Food intake was assessed by means of three 24-h dietary recalls applied on non-consecutive days. The mean energy and macronutrient intakes of each individual were calculated. Physical activity was evaluated using the long version of the international physical activity questionnaire (17) and reported as minutes per week.

Height was measured using a fixed stadiometer and weight was measured on a Filizola digital scale with the subjects wearing light clothing and no shoes. Body mass index (BMI) was subsequently calculated. Abdominal circumference was measured at the midpoint between the bottom of the rib cage and above the top of the iliac crest during minimal respiration. Percent body fat was estimated with a single-frequency bioimpedance analyzer (RJL Systems Inc., model Quantum II, USA). Tetrapolar placement of electrodes was used and individuals' weight, height, age and gender were entered into the instrument for analysis with the measured bioelectrical impedance to calculate body composition based on manufacturer equations.

Blood pressure was measured at rest in the sitting position, 3 times at 5-min intervals with an automatic blood pressure device (Omron HEM-712C, Omron Health Care, USA). The average of the last 2 recorded measurements was used for analysis.

Participants were submitted to a 75-g oral glucose tolerance test and venous blood samples were obtained for several determinations. Categories of glucose tolerance were defined according to American Diabetes Association criteria (15). Plasma glucose and lipoproteins were immediately determined in the local laboratory using routine methods. Aliquots were frozen at $-80^{\circ} \mathrm{C}$ for further determinations of inflammatory markers and hormones. Ultra-sensitive CRP, interleukin-6 (IL-6) and TNF- $\alpha$ were determined by an immunoenzyme chemiluminescent assay (Immulite, Diagnostic Products Corporation, USA). Insulin was determined by an immunometric assay using a quantitative chemiluminescent kit (AutoDelfia, Perkin Elmer Life Sciences Inc., USA) and adiponectin by an enzyme-linked immunosorbent assay (Linco Research, USA). Homeostasis model assessment was used to assess insulin resistance (HOMA-IR) (12). Insulin resistance was defined using the HOMA-IR cut-off value of 2.71 , based on a previous study conducted on a similar Brazilian sample (18).

\section{Statistical analysis}

Dietary, physical activity, clinical and inflammatory data are reported as means \pm SD or percentages. Nutrient intake is reported in grams and/or percent of total energy intake. Dietary data were adjusted according to the method of Willett and Stampfer (19) and processed using the Nutrition Data System ${ }^{\circledR}$ software (Minnesota Nutrition Coordinating Center, USA). Normality of variables was tested with the Kolmogorov-Smirnov test and those that were not normally 
distributed were log-transformed. The Student $t$-test (or a non-parametric equivalent) was used for comparisons between categories. Frequencies were compared by the chi-square test. Pearson's coefficient was used to test correlations between variables. In stepwise linear regression analysis, the HOMA-IR index was the dependent variable. Variables showing a $P$ value $<0.20$ in univariate analysis were selected for entry into the multiple model. Fatty acid intake was the independent variable of main interest, adjusted for age, gender, BMI, and total physical activity. Inflammatory markers (IL-6, adiponectin and CRP) were included to examine the influence of the inflammatory process on insulin resistance. HOMA-IR, trans fatty acid intake, physical activity, and adiponectin concentration were log-transformed.

All analyses were conducted using SPSS version 17.0 for Windows (SPSS Inc., USA). $A$ P value of $<0.05$ was considered to be significant.

\section{Results}

In the study sample of 183 individuals, 121 (66\%) were women, mean age was $55.7 \pm 12.3$ years and mean HOMA-IR values were $2.50 \pm$ 1.77. Participants were divided into 2 groups according to the cut-off value of HOMA-IR, with 127 individuals allocated to the group without insulin resistance and 56 to the group with insulin resistance (30.6\%). As expected, mean HOMA-IR values differed markedly between the groups with and without insulin resistance 4.49 \pm 1.88 vs $1.62 \pm 0.64, \mathrm{P}<0.001$, respectively) . Comparisons of mean dietary and clinical data are shown in Tables 1 and 2. Groups did not differ in energy or nutrient consumption. Insulinresistant individuals spent less time performing physical activity, had lower body adiposity, but similar blood pressure levels.

Mean HDL cholesterol and adiponectin values were lower, and IL-6 concentration was higher, in the insulin-resistant group compared to the group without insulin resistance (Table 2). Groups did not differ significantly concerning other laboratory variables.

Significant correlation coefficients were found between habits, anthropometric measurements, biomarkers, and HOMA-IR. Energy intake and abdominal circumference were correlated $(r=0.191, P=0.010)$. Energy $(r=-0.227$; $P=0.002)$ and omega- 6 polyunsaturated fatty acid $(r=-0.172, P=0.020)$ intakes were in-
Table 1. Dietary, physical activity, and clinical data of individuals stratified according to the presence of insulin resistance (IR).

\begin{tabular}{|c|c|c|}
\hline & Without IR (N = 127) & With IR $(N=56)$ \\
\hline \multicolumn{3}{|l|}{ Intake } \\
\hline Energy (kcal) & $1769.3 \pm 644.5$ & $1909.1 \pm 859.1$ \\
\hline Carbohydrate ${ }^{\#}(\mathrm{~g})$ & $210.6 \pm 32.8$ & $213.9 \pm 36.9$ \\
\hline $\operatorname{Protein}^{\#}(\mathrm{~g})$ & $77.6 \pm 18.5$ & $77.4 \pm 17.6$ \\
\hline Total fat ${ }^{\#}(\mathrm{~g})$ & $60.4 \pm 13.0$ & $59.3 \pm 13.6$ \\
\hline Saturated fat ${ }^{\#}(\mathrm{~g})$ & $18.8 \pm 6.2$ & $18.3 \pm 5.1$ \\
\hline Monounsaturated fat ${ }^{\#}(\mathrm{~g})$ & $20.9 \pm 5.7$ & $20.5 \pm 5.9$ \\
\hline Polyunsaturated fat ${ }^{\#}(\mathrm{~g})$ & $15.4 \pm 4.5$ & $15.6 \pm 4.4$ \\
\hline Omega 6 fatty acids ${ }^{\#}(\mathrm{~g})$ & $13.60 \pm 4.04$ & $13.66 \pm 3.97$ \\
\hline Omega 3 fatty acids ${ }^{\#}(\mathrm{~g})$ & $1.84 \pm 0.57$ & $1.99 \pm 0.67$ \\
\hline Omega 6/omega 3 ratio & $7.67 \pm 1.52$ & $7.45 \pm 1.15$ \\
\hline Trans fatty acids ${ }^{\#}(\mathrm{~g})$ & $3.25 \pm 2.23$ & $3.40 \pm 2.35$ \\
\hline Total fiber\# $(\mathrm{g})$ & $15.5 \pm 7.1$ & $14.6 \pm 5.7$ \\
\hline Total sugar $\#(g)$ & $75.1 \pm 25.0$ & $79.8 \pm 27.4$ \\
\hline Added sugar\# $(\mathrm{g})$ & $38.5 \pm 21.4$ & $39.8 \pm 22.2$ \\
\hline Total physical activity (min/week) & $228.3 \pm 239.3$ & $138.5 \pm 100.2^{*}$ \\
\hline Leisure physical activity (min/week) & $46.1 \pm 75.4$ & $19.9 \pm 41.6$ \\
\hline Body mass index $\left(\mathrm{kg} / \mathrm{m}^{2}\right)$ & $29.5 \pm 5.1$ & $33.9 \pm 6.1^{*}$ \\
\hline Abdominal circumference $(\mathrm{cm})$ & $97.9 \pm 11.0$ & $108.9 \pm 13.5^{*}$ \\
\hline Fat mass $(\%)$ & $32.8 \pm 8.8$ & $38.2 \pm 9.2^{*}$ \\
\hline Systolic blood pressure $(\mathrm{mmHg})$ & $135.5 \pm 20.3$ & $136.1 \pm 17.8$ \\
\hline Diastolic blood pressure $(\mathrm{mmHg})$ & $82.2 \pm 10.3$ & $84.0 \pm 10.3$ \\
\hline
\end{tabular}

Data are reported as means \pm SD. \#Adjusted according to Willett and Stampfer (19). ${ }^{*} P<0.05$ compared to without IR (Student $t$-test for independent samples).

Table 2. Laboratory variables of individuals stratified according to the presence of insulin resistance (IR).

\begin{tabular}{lcc}
\hline & Without IR $(\mathrm{N}=127)$ & With IR $(\mathrm{N}=56)$ \\
\hline Fasting plasma glucose $(\mathrm{mg} / \mathrm{dL})$ & $96.9 \pm 11.3$ & $104.5 \pm 10.2^{*}$ \\
Post-load plasma glucose $(\mathrm{mg} / \mathrm{dL})$ & $115.0 \pm 26.9$ & $125.8 \pm 27.3^{*}$ \\
Triglycerides $(\mathrm{mg} / \mathrm{dL})$ & $144.3 \pm 60.7$ & $165.9 \pm 82.7$ \\
Fasting insulin $(\mu \mathrm{lU} / \mathrm{mL})$ & $6.89 \pm 2.87$ & $17.60 \pm 7.48^{*}$ \\
Total cholesterol $(\mathrm{mg} / \mathrm{dL})$ & $202.1 \pm 37.9$ & $192.2 \pm 50.6$ \\
HDL cholesterol $(\mathrm{mg} / \mathrm{dL})$ & $43.4 \pm 11.5$ & $39.6 \pm 11.9^{*}$ \\
LDL cholesterol $(\mathrm{mg} / \mathrm{dL})$ & $129.0 \pm 35.2$ & $119.2 \pm 45.0$ \\
VLDL cholesterol $(\mathrm{mg} / \mathrm{dL})$ & $28.5 \pm 11.3$ & $32.2 \pm 15.0$ \\
Leukocytes $\left(\mathrm{N} / \mathrm{mm}{ }^{3}\right)$ & $6606 \pm 1744$ & $7065 \pm 1745$ \\
Interleukin-6 $(\mathrm{pg} / \mathrm{mL})$ & $2.77 \pm 3.10$ & $3.79 \pm 3.33^{*}$ \\
C-reactive $\mathrm{protein}(\mathrm{mg} / \mathrm{dL})$ & $0.48 \pm 0.52$ & $0.60 \pm 0.59$ \\
Adiponectin $(\mathrm{ng} / \mathrm{mL})$ & $16.2 \pm 15.4$ & $11.3 \pm 6.9^{*}$ \\
TNF- $\alpha(\mathrm{ng} / \mathrm{mL})$ & $12.5 \pm 6.5$ & $12.4 \pm 7.1$ \\
\hline
\end{tabular}

Data are reported as means \pm SD. TNF- $\alpha=$ tumor necrosis factor alpha. ${ }^{*} \mathrm{P}<0.05$ compared to without IR (Student $t$-test for independent samples). 

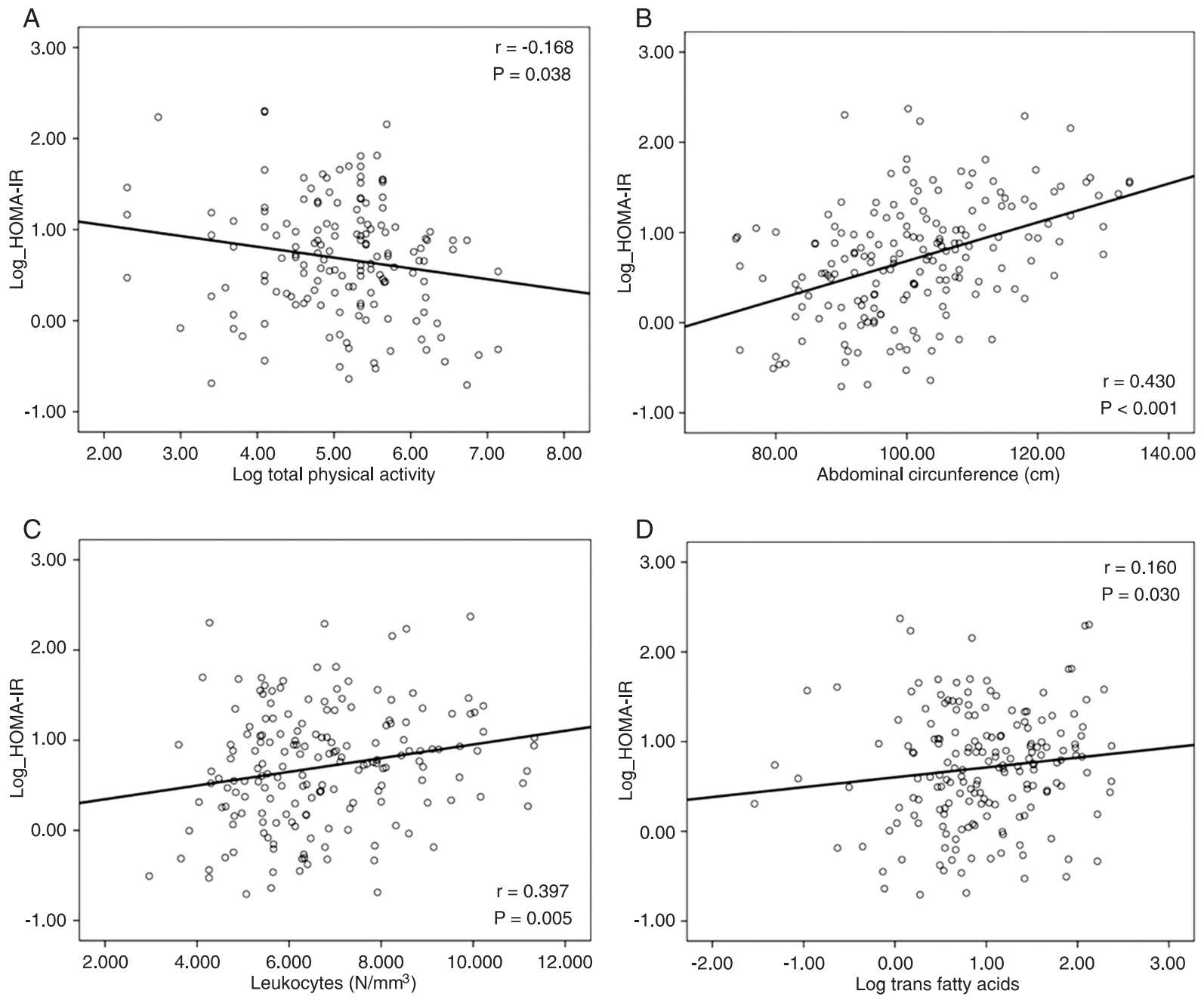

Figure 1. Correlation of HOMA-IR with total physical activity (Panel A), abdominal circumference (Panel B), leukocyte count (Panel $C)$, and trans fatty acid intake $($ Panel $D)$. HOMA-IR = homeostasis model assessment-insulin resistance.

versely correlated with adiponectin concentration. Saturated fat intake was correlated with IL-6 levels $(r=0.172, P=0.025)$, leukocyte count $(r=0.153$, $P=0.041)$, and added sugar to CRP $(r=0.210, P$ $=0.005)$. Fat mass also showed a correlation with CRP ( $r=0.419, \mathrm{P}<0.001$; Figure 1, Panel B).

HOMA-IR was correlated with several variables (Figure 1): physical activity $(r=-0.168, P=0.038)$, trans fatty acid intake $(r=0.160, P=0.030)$, abdominal circumference $(r=0.430, P<0.001)$, leukocyte count $(r=0.397, P=0.005)$, and adiponectin concentrations $(r=-0.288, P<0.001)$.

Independent associations with HOMA-IR in multiple linear regression analysis are shown in Table 3. The final model revealed that HOMA-IR was independently associated with trans fatty acid intake $(\beta=1.416, P=$ IR.
Table 3. Final linear regression model for the association with HOMA-

\begin{tabular}{lrcr}
\hline & $\beta$ & $95 \%$ confidence interval & \multicolumn{1}{c}{$\mathrm{P}$} \\
\hline Trans fatty acid intake $^{\#}$ & 1.416 & 1.016 to 1.820 & 0.039 \\
Adiponectin $^{\#}$ & -1.637 & -2.203 to -1.164 & 0.004 \\
Age & 0.051 & -0.002 to 0.005 & 0.510 \\
Physical activity & -1.180 & -1.426 to 1.125 & 0.325 \\
Body mass index & 0.390 & 0.012 to 0.026 & $<0.001$ \\
\hline
\end{tabular}

\#Anti-log-transformed values. HOMA-IR = homeostasis model assessment-insulin resistance.

$0.039)$ and $\mathrm{BMI}(\beta=0.390, P<0.001)$, and also inversely associated with adiponectin $(\beta=-1.637, P=0.004)$, after 
adjustment for age, gender and physical activity. Inclusion of other nutrients such as saturated fat or added sugar or other inflammatory markers (IL-6 and CRP) in the models did not modify these associations.

\section{Discussion}

The present study contributes to the knowledge about the pathophysiologic relationship between certain dietary habits - particularly fatty acid intake - and insulin resistance. HOMA-IR was used to express insulin resistance and its cut-off value was obtained in a Brazilian sample with similar sociodemographic and clinical characteristics as the present sample (18). The cut-off of 2.71 does not differ markedly from others previously proposed (20). Since our study sample had risk factors for type 2 diabetes mellitus, a considerable proportion of insulin-resistant individuals was expected $(30.6 \%)$. Several findings indicate that this value is adequate to identify insulin resistance; this subset showed higher body adiposity and plasma concentrations of glucose and IL-6, as well as lower concentrations of HDL cholesterol and adiponectin, typical abnormalities found in metabolic syndrome.

The independent association of trans fatty acid intake and HOMA-IR was statistically significant in our study, although the clinical relevance of this finding requires further investigation. The deleterious role of saturated and trans fatty acids in glucose and lipid metabolism has been consistently demonstrated $(3,4,7)$. This effect is due at least in part to increased body fatness, since hypertrophic adipocytes and infiltrating macrophages are important sources of cytokines - such as TNF- $\alpha$ and IL- 6 - which cause the deterioration of insulin sensitivity. The total fat intake found in our sample (approximately $30 \%$ of total energy intake) is comparable to levels reported in other Western populations (21). In crude analysis, total fat intake was not correlated with body adiposity, although the latter was shown to be associated with inflammatory markers (percent of fat mass with CRP) and insulin resistance (abdominal circumference with HOMA-IR). Interestingly, saturated fat consumption seemed to be correlated with IL-6 and leukocyte count, a fact that was not confirmed after adjustment. The ability of certain nutrients, such as fatty acids, to penetrate through the lipoprotein membrane and to induce gene expression has been clearly demonstrated $(7,22)$.

This set of findings is coherent with the statement that obesity is a condition involving inflammation and insulin resistance. Evidence from animal models has shown that inflammatory cytokines cause intracellular abnormalities such as activation of IkB kinase (IKK) and c-Jun N-terminal kinase (JNK) and increased serine phosphorylation of insulin receptor substrate 1 , which inhibit insulin signaling and glucose transport, generating insulin resistance $(23,24)$. Our data may suggest that these processes, inflammation and insulin resistance, coexist in the participants of this study.
A direct correlation of HOMA-IR with leukocyte count and an inverse correlation of HOMA-IR with adiponectin are consistent with the hypothesis that intracellular inflammatory factors contribute to inducing insulin resistance $(3,16)$. Low circulating levels of adiponectin have been described within the spectrum of metabolic syndrome (25). Although associations with pro-inflammatory factors were not supported by our study, the detection of an independent inverse association of HOMA-IR with adiponectin is relevant considering its cardioprotective effect.

Using multiple linear regression, we investigated whether saturated and trans fatty acids, independent of their impact on body adiposity, could contribute to the generation of inflammation and the deterioration of insulin sensitivity. In animal models, a high-fat diet induces endoplasmic reticulum stress, which activates IKK and JNK, thereby impairing insulin signaling (24). The gut microbiota of obese individuals or those consuming a high content of these fatty acids contains a predominance of Gram-negative bacteria rich in lipopolysaccharides (LPS). LPS are recognized by Toll membrane receptors in the circulation (endotoxemia), activating these kinases to generate insulin resistance, as well as NFKB, which result in the expression of inflammatory genes. Similar to LPS, saturated fatty acids are also recognized by membrane receptors triggering proinflammatory signaling pathways (26).

A strength of the present study was the availability of physical activity data; this practice could introduce a potential confounder in the analysis of the relationship of dietary factors and metabolic abnormalities. Exercise enhances glucose uptake by stimulating glucose transporter type 4 translocation to the plasma membrane, independently of insulin (27). However, the level of physical activity was not associated with HOMA-IR in the present study. This lack of association may be attributed at least in part to the low frequency of physical activity practiced by our sample. Adjustment for physical activity did not alter the results of HOMA-IR with nutrients obtained from the regression analysis.

The crude analysis seemed to support previous evidence that saturated fatty acid intake could induce a proinflammatory status, but in adjusted analysis only trans fatty acids were independently associated with insulin resistance. Considering that foods rich in trans fat may have a high sugar content, we tested whether the inclusion of added sugar could be a confounder in our analysis of the association with HOMA-IR. However, added sugar proved not to influence the strength of the association of interest.

Considering the hypothesis that fatty acid intake may impair inulin sensitivity indirectly via the expression of inflammatory genes (TNF- $\alpha$ and interleukins), IL-6 or CRP and adiponectin concentrations were selected for input to the regression model. Adiponectin remained inversely associated with HOMA-IR, a finding consistent with its expected protective effects (2), but IL-6 or CRP did not. 
Since inflammatory markers did not modify the association of trans fatty acid intake with HOMA-IR, our results did not support the hypothesis that trans fatty acids can generate insulin resistance by inducing proinflammatory cytokine expression. These findings conflict with the results of other studies showing that NF-kB is activated by trans fatty acid (28).

Our study has limitations. First, the design is not appropriate to investigate causal relationships and the rate of losses was relatively high. Second, there was a limitation inherent to the tool used for assessing diet; despite extensive use of 24-h food recalls, three recalls may not be sufficiently sensitive to quantify nutrient intake; additionally, insulin resistance develops over the long-term while food recalls reflect recent habits. This aspect may have precluded the

\section{References}

1. Kannel WB, Belanger A, D'Agostino R, Israel I. Physical activity and physical demand on the job and risk of cardiovascular disease and death: the Framingham Study. Am Heart J 1986; 112: 820-825.

2. Fonseca-Alaniz MH, Takada J, Alonso-Vale MI, Lima FB. [The adipose tissue as a regulatory center of the metabolism]. Arq Bras Endocrinol Metabol 2006; 50: 216-229.

3. Kennedy A, Martinez K, Chuang CC, LaPoint K, Mclntosh $M$. Saturated fatty acid-mediated inflammation and insulin resistance in adipose tissue: mechanisms of action and implications. J Nutr 2009; 139: 1-4.

4. Mozaffarian D. Trans fatty acids - effects on systemic inflammation and endothelial function. Atheroscler Suppl 2006; 7: 29-32.

5. Brasil MdSS. Sistema de Informação sobre Mortalidade. Porcentagem de óbitos segundo grupo de causas. Available at [http://tabnet.datasus.gov.br/cgi/tabcgi.exe?idb2006/c04. def].

6. Uemura K, Pisa Z. Recent trends in cardiovascular disease mortality in 27 industrialized countries. World Health Stat $Q$ 1985; 38: 142-162.

7. Saravanan N, Haseeb A, Ehtesham NZ, Ghafoorunissa. Differential effects of dietary saturated and trans-fatty acids on expression of genes associated with insulin sensitivity in rat adipose tissue. Eur J Endocrinol 2005; 153: 159-165.

8. Thompson AK, Minihane AM, Williams CM. Trans fatty acids, insulin resistance and diabetes. Eur J Clin Nutr 2011; 65: 553564.

9. Peres SB, de Moraes SM, Costa CE, Brito LC, Takada J, Andreotti $S$, et al. Endurance exercise training increases insulin responsiveness in isolated adipocytes through IRS/ PI3-kinase/Akt pathway. J Appl Physiol 2005; 98: 10371043.

10. International Diabetes Federation. Diabetes Atlas. 3rd edn. Brussels: International Diabetes Federation; 2006.

11. Pineo CE, Anderson JJB. Cardiovascular benefits of the Mediterranean diet. Nutrition Today 2008; 43: 114-120.

12. Matthews DR, Hosker JP, Rudenski AS, Naylor BA, Treacher DF, Turner RC. Homeostasis model assessment: insulin resistance and beta-cell function from fasting plasma glucose detection of the previously reported association of saturated fat intake with insulin resistance (7).

The finding of an association of trans fatty acid intake with HOMA-IR corroborates the assumption that this nutrient could play a role in the generation of insulin resistance, but not necessarily by stimulating inflammatory genes. We speculate that trans fatty acids should predominantly influence insulin sensitivity via activation of intracellular kinases, which alter insulin receptor substrates. However, activation of transcription factors for the expression of proinflammatory genes, contributing to reduced glucose uptake, cannot be excluded. Studies with a suitable design and more accurate tools for measuring long-term food consumption are desirable to refine future investigations of these associations.

and insulin concentrations in man. Diabetologia 1985; 28 : 412-419.

13. Shah A, Mehta N, Reilly MP. Adipose inflammation, insulin resistance, and cardiovascular disease. J Parenter Enteral Nutr 2008; 32: 638-644.

14. Thom T, Haase N, Rosamond W, Howard VJ, Rumsfeld J, Manolio T, et al. Heart disease and stroke statistics - 2006 update: a report from the American Heart Association Statistics Committee and Stroke Statistics Subcommittee. Circulation 2006; 113: e85-e151.

15. Report of the Expert Committee on the Diagnosis and Classification of Diabetes Mellitus. Diabetes Care 1997; 20: 1183-1197.

16. Hotamisligil GS, Shargill NS, Spiegelman BM. Adipose expression of tumor necrosis factor-alpha: direct role in obesity-linked insulin resistance. Science 1993; 259: 8791.

17. Craig CL, Marshall AL, Sjostrom M, Bauman AE, Booth ML, Ainsworth BE, et al. International physical activity questionnaire: 12-country reliability and validity. Med Sci Sports Exerc 2003; 35: 1381-1395.

18. Geloneze B, Repetto EM, Geloneze SR, Tambascia MA, Ermetice $M N$. The threshold value for insulin resistance (HOMA-IR) in an admixtured population IR in the Brazilian Metabolic Syndrome Study. Diabetes Res Clin Pract 2006; 72: $219-220$.

19. Willett W, Stampfer MJ. Total energy intake: implications for epidemiologic analyses. Am J Epidemiol 1986; 124: 17-27.

20. Oliveira EP, Souza MLA, Lima MDA. Índice HOMA (homeostasis model assessment) na prática clínica: uma revisão. $J$ Bras Patol Med Lab 2005; 41: 237-243.

21. World Health Organization (WHO). Diet, nutrition and the prevention of chronic diseases. Report of a joint WHO/FAO expert consultation. Geneva: WHO Technical Report Series, 916; 2003.

22. Shaikh SR, Edidin M. Polyunsaturated fatty acids, membrane organization, T cells, and antigen presentation. Am J Clin Nutr 2006; 84: 1277-1289.

23. Fernandez-Veledo S, Vila-Bedmar R, Nieto-Vazquez I, Lorenzo M. c-Jun N-terminal kinase $1 / 2$ activation by tumor 
necrosis factor-alpha induces insulin resistance in human visceral but not subcutaneous adipocytes: reversal by liver $X$ receptor agonists. J Clin Endocrinol Metab 2009; 94: 35833593.

24. Tobar N, Oliveira AG, Guadagnini D, Bagarolli RA, Rocha GZ, Araujo TG, et al. Diacerhein improves glucose tolerance and insulin sensitivity in mice on a high-fat diet. Endocrinology 2011; 152: 4080-4093.

25. Aprahamian TR, Sam F. Adiponectin in cardiovascular inflammation and obesity. Int J Inflam 2011; 2011: 376909.

26. Tsukumo DM, Carvalho BM, Carvalho-Filho MA, Saad MJ.
Translational research into gut microbiota: new horizons in obesity treatment. Arq Bras Endocrinol Metabol 2009; 53: 139-144.

27. Cartee GD, Wojtaszewski JF. Role of Akt substrate of 160 $\mathrm{kDa}$ in insulin-stimulated and contraction-stimulated glucose transport. Appl Physiol Nutr Metab 2007; 32: 557-566.

28. Bryk D, Zapolska-Downar D, Malecki M, Hajdukiewicz K, Sitkiewicz D. Trans fatty acids induce a proinflammatory response in endothelial cells through ROS-dependent nuclear factor-kappaB activation. J Physiol Pharmacol 2011; 62: 229-238. 